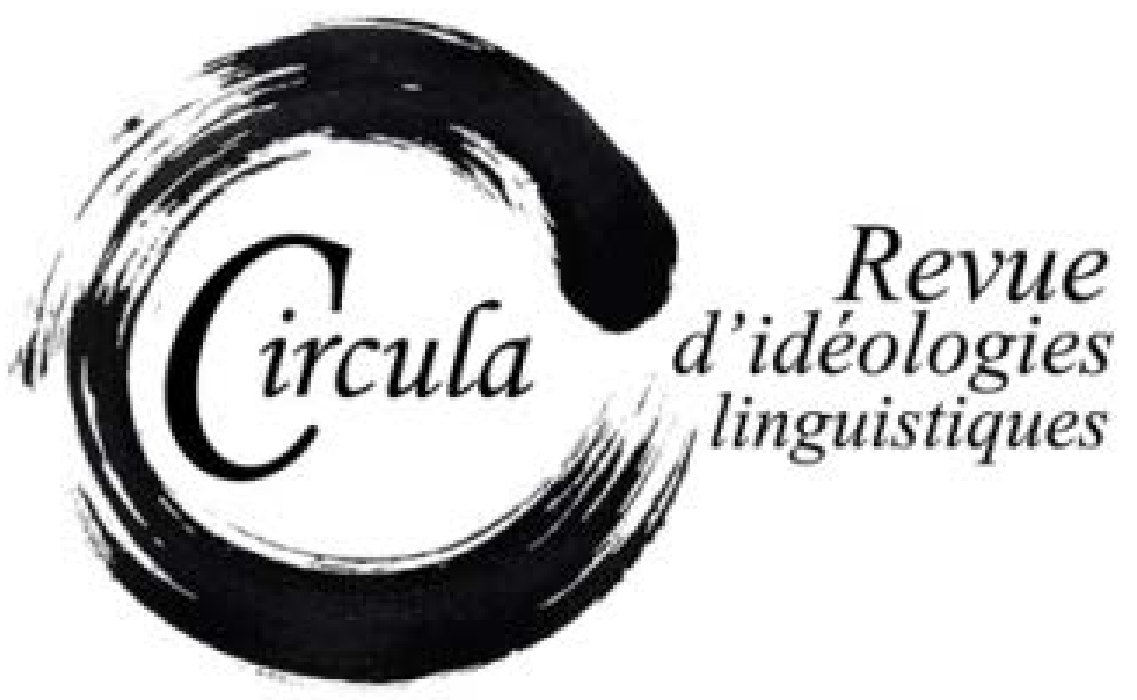

TITRE: LA SATIRE DES MOTS À LA MODE DANS LA PRESSE FRANÇAISE : IDÉOLOGIE LINGUISTIQUE ET IDÉOLOGIE POLITIQUE

Auteur(s): Agnès Steuckardt, Université PAul-VAlÉry-MontPelLier

Revue: Circula, NumÉRO 1, PAGES 26-43

ISSN: 2369-6761

DiRECTEURS: Wim REMYSEN ET SABINE SCHWARZE

URI: HTTP://HDL.HANDLE.NET/11143/8000

DOI: $10.17118 / 11143 / 8000$ 


\section{La satire des mots à la mode dans la presse française : idéologie linguistique et idéologie politique}

Agnès Steuckardt, Université Paul-Valéry-Montpellier

agnes.steuckardt @ univ-montp3.fr

Résumé : La satire des mots à la mode s'inscrit dans une longue tradition française. Elle entre dans la presse pendant la Révolution : les journalistes contre-révolutionnaires se moquent des néologismes imaginés par les révolutionnaires. Sans être l'apanage de la droite, elle présente des affinités avec un conservatisme politique dont témoignent, à la fin du XXe siècle, les chroniques puristes du Figaro. Depuis 2008 cependant, Libération puis, à partir de 2011, Le Monde proposent des chroniques de langue d'une orientation sensiblement différente. Les usagers des mots à la mode y apparaissent comme les victimes inconscientes d'une novlangue venue de l'entreprise et des milieux de la communication. La satire des mots à la mode entre ainsi dans un schéma nouveau : ce n'est plus la droite qui reproche à la gauche d'abuser d'un jargon à la mode, mais plutôt la gauche qui raille un jargon à la mode initié par la droite.

Mots-clés : néologisme; jargon; mot à la mode ; presse ; gauche/droite

Abstract: The satire of buzzwords is part of a long French tradition. This phenomenon appears in the press during the French Revolution: counterrevolutionary journalists make fun of neologisms created by revolutionaries. Although not exclusivy used by right-wing journals, it has affinities with political conservatism shown by the purist chronicles of Figaro at the end of the 20th century. Since 2008, however, Liberation and, from 2011, Le Monde offer language columns of a substantially different orientation. Users of buzzwords appear as unconscious victims of Newspeak from the business and communication world. The satire of buzzwords enters a new paradigm: once the right criticized the left because it was abusing a fashionable jargon, today the left mocks a fashionable jargon created by the right.

Keywords: neologism; jargon; buzzword; press; left/right 


\section{Introduction}

La satire des mots à la mode a-t-elle une couleur politique ? Cette activité, qui relève par nature de ce qu'on appelle volontiers aujourd'hui la « linguistique populaire », et plus précisément - par le jugement négatif qu'elle porte sur une forme de l'innovation langagière - de l'idéologie linguistique, relève-t-elle aussi de l'idéologie politique? Se moquer des mots à la mode, est-ce de droite, est-ce de gauche?

La légitimité même de la question peut être mise en doute : « La division droite-gauche a-t-elle un sens en dehors de la politique? » se demandait René Rémond (1982:381). Il tranchait par l'affirmative, en s'appuyant en particulier sur une observation qui touche la langue française, et sa problématique mobilité :
Il est notoire qu'un écrivain de droite a plus de chance d'entrer un jour sous la Coupole qu'un écrivain de gauche ; au reste un écrivain de gauche ne tentera pas sa chance. C'est ainsi que l'Académie française fait figure de club conservateur. (Rémond, 1982 : 382)

Même si l'on admet la légitimité de la question, la définition de la droite et de la gauche étant ellemême éminemment fluctuante, il serait aventuré d'espérer y répondre selon une dichotomie tranchée.

Si l'on se rapporte cependant aux origines des notions de droite et de gauche, on peut souligner que, dans le contexte de la Révolution française, qui les a vu naître, la satire des mots à la mode a été essentiellement le fait des contre-révolutionnaires, et donc qu'elle est, au moins par cette origine, ancrée à droite ${ }^{1}$. On voudrait remarquer ici une tendance relativement récente qui semble déporter vers la gauche la satire des mots à la mode. On en prendra pour indice le constat factuel suivant : alors que de 1996 à 2006, c'était dans Le Figaro que l'on pouvait lire des chroniques linguistiques épinglant les mots à la mode, depuis 2008, c'est dans les colonnes de Libération, puis du Monde qu'on a pu trouver des satires de ce type. Au vu de ces faits, on formulera l'hypothèse que la satire des mots à la mode s'est déplacée, au moins pour partie, d'une idéologie de droite vers une idéologie de gauche, ou du moins de centre gauche.

Pour explorer cette piste, on reviendra dans un premier temps sur l'association traditionnelle entre satire des mots à la mode et idéologie de droite. On montrera ensuite, à partir de deux corpus de presse, l'un tiré du journal Libération pendant la période juillet-août 2008, l'autre du journal Le Monde de mars 2011 à mars 2014, comment deux journaux idéologiquement ancrés respectivement à gauche et au centre gauche peuvent aujourd'hui porter ce type de discours.

1. Le genre de la «chronique de langage » n'est pas constitué en tant que tel dans la presse révolutionnaire. On s'appuie dans la présente étude sur les remarques linguistiques que l'on peut relever dans les journaux contre-révolutionnaires L'année littéraire et politique (Royou) et Les Actes des Apôtres d'une part, et d'autre part sur celles d'un proche de Mirabeau, Antoine Cérutti, qui contribua à plusieurs journaux (le Journal de Paris, la Feuille villageoise). 


\section{Un ancrage originel à droite}

La notion de « mot à la mode», et la réaction critique que ces mots déclenchent, remontent probablement à la plus haute antiquité : chez les Romains par exemple, la mode des hellénismes s'attirait les critiques acerbes du poète-moraliste Juvénal². Plus généralement, les grammairiens et rhéteurs traditionnalistes de l'antiquité latine - Varron, Aulu-Gelle, Quintilien - regardaient les mots nouveaux comme un danger (Griffe, 2001 : 131-156). Nous nous intéresserons plus précisément ici à l'ancrage idéologique de cette notion dans l'aire culturelle française.

\subsection{Satire des mots à la mode et stabilité sociale}

Au XVI e siècle, la propension des courtisans à farcir leur conversation d'italianismes suscite la colère d'un Henri Estienne, qui publie contre cette pratique un mordant pamphlet : les Deux Dialogues du nouveau langage françois italianizé (1578). Si la satire des mots à la mode est déjà effective, l'expression de mot(s) à la mode n'existe pas encore. Elle se diffusera au milieu du XVII siècle, pour nommer et stigmatiser les usages linguistiques des Précieuses : Gilles Siouffi la relève chez l'Abbé de Pure dans La Prétieuse ou le Mystere des ruelles (1656; Siouffi, Steuckardt et Wionet, 2014). Une requête dans le Grand Corpus des grammaires, remarques et traités de langue (XIVe-XVII siècles) permet de repérer la récurrence des énoncés ce mot est fort à la mode et ces mots sont fort à la mode chez les grammairiens et remarqueurs du XVII e siècle ${ }^{3}$. À la fin du XVII ${ }^{e}$ siècle, la lexie s'installe dans un usage moins spécialisé avec, en 1692, l'ouvrage de François de Callières intitulé Les mots à la mode, et, en 1694, la pièce de Boursault du même nom. Il serait bien sûr anachronique de situer ces textes idéologiquement à droite ou à gauche. On ne s'interdira pas toutefois d'en risquer une lecture sociopolitique. La question des mots à la mode s'inscrit d'emblée dans une problématique sociologique : elle oppose la vieille noblesse d'épée aux jeunes courtisans chez Callières, la bourgeoisie assumée contre la bourgeoisie à prétention nobiliaire chez Boursault (d'après le modèle mis en place par Molière dans les Précieuses ridicules ou Les Femmes savantes). Selon leurs détracteurs, les mots à la mode constituent un dissensus interne à la classe ${ }^{4}$ : ils clivent la noblesse chez Callières, la bourgeoisie

\footnotetext{
2. La Satire III, par exemple, se moque des ornements vestimentaires que croit devoir porter tel paysan romain pour suivre la mode et des noms grecs qui les désignent : « Rusticus ille tuus sumit trechidipna, Quirine,/Et ceromatica collo fert niceteria collo » ("Ce paysan dont tu es l'ancêtre, Quirinus,/Chausse des trechedipnes [hapax: "sandales"] et porte des niceteries [hapax: "médailles" ?] autour de son cou céromatiqué [hapax, "huilé"] »), Satire III, v. 67-68, [II siècle apr. J.-C.] 1965 : 54).

3. Par exemple, dans cette remarque de Vaugelas à propos des préfixés en super: « Ces mots au reste composez de cette particule super étoient fort à la mode autrefois. On disoit superabondant, superabondance, supernaturel, supernaturellement, supernel, supernellement, supernumeraire, superscription, superseder. Mais on ne souffre plus guéres aujourd'huy ce super dans nos termes. Car nous disons surabondant, surabondance, surabondamment, surnaturel, surnaturellement, surnumeraire, \& suscription » (Vaugelas, 1690 : 215-216). Dans le Grand corpus des grammaires françaises, des remarques et des traités sur la langue (XIVe-XVIIe siècles), la locution à la mode présente 79 occurrences, dont 31 portent sur les phénomènes lexicaux, prosodiques ou stylistiques.
}

4. «On pourrait conclure que le livre précédent [Les Mots à la mode] a, au moins fictivement, réussi à rassembler - ou à 
chez Molière ou Boursault. La satire des mots à la mode signale alors une idéologie sociopolitique conservatrice, les adversaires des mots à la mode se présentant comme les défenseurs d'une unité et d'une stabilité du groupe social.

Au début du XVIII' siècle, le genre de la satire linguistique prospère, notamment avec l'invention du qualificatif néologique, imaginé par l'abbé Desfontaines pour le titre d'un ouvrage destiné à railler les innovations lexicales et sémantiques des «modernes » : le Dictionnaire néologique, publié en 1726 (Steuckardt, 2011). Au cours du siècle cependant, les débats sur la langue se déplacent.

Le thème de l'abus des mots, philosophico-politique, devient central. Ce ne sont pas, dans le discours des philosophes, les mots nouveaux qui se trouvent dénoncés, mais des mots qu'ils jugent vagues ou porteurs de préjugés sociaux, tels bassesse et honnêtes gens analysés par Diderot dans l'Encyclopédie des Sciences et des arts (Ricken, 1982 ; Steuckardt, 2001, 2008). Parallèlement, les Encyclopédistes diffusent des mots créés dans le domaine des sciences et techniques, et des écrivains comme Restif de la Bretonne et Louis-Sébastien Mercier se font les promoteurs de la néologie. La faveur grandissante pour les mots nouveaux estompe le marquage péjoratif de néologique : son trait dépréciatif s'affaiblit, il devient un terme neutre, de langue de spécialité. Moins linguistique et plus sociale, la lexie mots à la mode continue à porter une appréciation négative sur les innovations linguistiques du moment, mais le genre de la satire des mots à la mode passe, pour ainsi dire, de mode.

\subsection{Satire des mots à la mode et contre-révolution}

L'événement révolutionnaire donne un sens nouveau aux prises de position linguistiques : la satire des mots à la mode y prend une signification politique. L'afflux de nouveaux mots et tours suscite en effet la raillerie des contre-révolutionnaires. Le thème de l'abus des mots, utilisé au long du XVIIIe siècle pour saper le vocabulaire d'ancien régime, est alors retourné contre les porteurs des idées politiques nouvelles. Au début de la Révolution, de petits opuscules comme L'Abus des mots (1789) ou Les Synonymes nouveaux (1789) attaquent les mots nouvellement diffusés tels que aristocrate, ou nation. La presse contre-révolutionnaire s'empare du thème. Ainsi Les Actes des Apôtres, journal contre-révolutionnaire dont l'un des rédacteurs est Rivarol, se gaussent-il du terme aristocrassique, entraîné par la vogue d'aristocrate:

rappeler à l'ordre de leur habitus social - les protagonistes nobles. Incités par le Commandeur à délaisser la mode, qui divisait leur propre groupe, ils montreraient, dans ce nouvel ouvrage, comment partager un seul usage, lequel pourrait s'imposer comme norme légitime aux bourgeois dominés. » (Merlin-Kajman, à paraître) 
Les aristocrates ont répété avec une joie indécente que, le Jeudi, 19 novembre, M. de Robespierre, dans la chaleur de la discussion sur la démarche du Bureau renforcé du Cambresis, avait dit que ce Bureau était un corps aristocrassique, que l'esprit aristocrassique dirigeait uniquement, et qu'il fallait s'empresser de le détruire. Le mot aristocrassique fit sourire les Auditeurs; cependant l'érudition, le goût, et les talents de M. de Robespierre, qui l'ont conduit à la Tribune nationale, sont connus de toute le France. Si le despotisme d'un pédant de Collége ne tolère pas un solécisme, la liberté de l'Assemblée doit souffrir parfois une expression qui s'éloigne si peu de la pureté du langage. (Les Actes des Apôtres, 1789 : 9-10)

La dérivation aristocrassique produit avec le mot crasse une fâcheuse consonance, dont l'effet immédiat est de «faire sourire les Auditeurs » et l'effet différé de susciter l'ironie du journaliste anonyme, qui utilise l'antiphrase pour saluer le « goût » de Robespierre, et la litote pour qualifier une expression «qui s'éloigne si peu de la pureté du langage ». De même que cette variation sur le mot honni d'aristocrate, le sur-emploi des « mots chéris » (Brunot, 1937 : 636) fait l'objet d'attaques satiriques. On lit par exemple dans L'année littéraire et politique de l'abbé Royou (continuation de L'Année littéraire de Fréron), à propos de la tragédie Charles IX ou l'Ecole des rois du révolutionnaire Marie-Joseph Chénier, les critiques suivantes:

J'avoue que je ne sais ni comment ni pourquoi on donne le nom de patriotique à la peinture de crimes bas et atroces de la cour de Charles IX. Mais ce mot de patriotique, ainsi que celui de national sont devenus à la mode et s'appliquent indistinctement aux objets auxquels ils ont le moins de rapport, au grand regret des bons citoyens qui gémissent de voir prodiguer, et, en quelque sorte, profaner des termes aussi respectables. (L'année littéraire et politique, $n^{\circ} 12$, avril 1790, p. 194-195)

Dans cette conjoncture historique qui présente un caractère fondateur pour la société française, la satire des mots « devenus à la mode » tels que national et patriotique rencontre l'idéologie politique contre-révolutionnaire, que l'on peut aussi qualifier d'idéologie politique « de droite », la notion de «droite » émergeant précisément dans l'événement révolutionnaire.

Les notions de droite et de gauche s'élaborent dans le contexte spatial de la salle des Menus Plaisirs à Versailles, lors du débat sur le droit de veto du Roi. La configuration idéologico-spatiale sera conservée lorsque l'Assemblée s'installe dans la salle du Manège le 19 octobre à Paris. Le côté droit était celui du veto absolu, et donc des députés les plus conservateurs. De cette origine, la notion de « droite » garde, dans sa définition, une orientation vers le conservatisme, ainsi que le résume à grands traits Michel Winock:

La droite veut préserver ce qu'elle est en mesure de sauver de l'héritage du passé, de la tradition, de la coutume : stabilité d'abord! La gauche n'a d'yeux que pour l'avenir, à construire de toutes pièces éventuellement : changement d'abord! L'une est conservatrice, l'autre progressiste. (Winock, 2012: 7) 
La constitution historique de la droite semble la placer en position d'adversaire naturel des mots à la mode, et le combat linguistique mené par des journalistes tels que l'abbé Royou tend à confirmer cette représentation.

\subsection{Autodérision linguistique à gauche}

Il faut néanmoins nuancer d'emblée cette homologie entre idéologie politique et idéologie linguistique, car, dès les débuts de la Révolution, la satire des mots à la mode a pu trouver place sous la plume d'écrivains de gauche, comme Chantreau:

MODE : dans l'ancien régime, où l'on ne s'occupoit que de superfluités, c'étoit la mode, et les modes françaises étoient les modes de l'univers. On en rafolloit même à Vienne, à Berlin, à Madrid, à Naples, à Turin sur-tout, etc., etc. Aujourd'hui que nous avons brisé les pompons, et que sur les pots à rouge on lit végétal national, au lieu de la mode nous disons le mode, un mode de gouvernement, fixer un mode pour la perception de tel impôt, etc. (Chantreau, 2008 [1790] : 126-127)

Le très révolutionnaire Chantreau se montre capable de rire des usages linguistiques que l'événement révolutionnaire a mis à la mode. L'homonymie entre la mode et le mode permet un jeu de mot qui fonde le développement plaisant de l'article : à "la mode », associée aux valeurs d'une monarchie décadente, le discours politique révolutionnaire préfère «le mode », dont il use dans les expressions citées, et bien d'autres, comme l'indique le « etc. ». Au-delà de cette substitution significative, Chantreau se moque de la transposition de la terminologie politique dans des domaines inattendus : le vocabulaire politique lui-même est «à la mode » et va se loger jusque sur les « pots à rouge », la pâte de maquillage étant rebaptisée végétal national. Un journaliste comme Antoine Cérutti inscrit dans la presse un discours satirique du même type (Baecque, 2000 ; Guilhaumou, 2009). Donnons-en une illustration:

Pour exprimer une émotion légère qui n’avertiroit personne, servez-vous du mot commotion électrique qui frappera tout le monde. Au lieu de dire que les esprits sont en effervescence, chose qui est trop commune et ne représente qu'une chaleur obscure, dites qu'ils sont en incandescence, ce qui est le dernier terme de la chaleur physique, et semble environner de rayons toutes les têtes. (Micromégas [Antoine Cérutti], [1789] : 50)

Sous la forme d'un « ne dites pas... mais dites » ironique, Cérutti se moque de la mode des métaphores scientifiques et du style emphatique dans l'éloquence révolutionnaire. La satire des mots à la mode qui exprimait au XVII ${ }^{\text {e }}$ iècle un clivage interne à une classe sociale, prend avec la Révolution une signification plus clairement politique, en s'inscrivant dans un antagonisme gauche/droite. Cette activité épilinguistique n'est pas donc l'apanage de la droite, et ce dès la Révolution, mais elle entretient, par sa nature conservatrice, des affinités particulières avec la droite. 
Il n'est pas possible, dans le cadre de cette étude, de suivre les inflexions politiques du discours sur les mots à la mode dans la presse des XIX et XXe siècles : l'enquête menée ici interroge la continuité de cet ancrage initial à droite dans la presse contemporaine. Les chroniques de langage dans un journal contemporain situé à droite, Le Figaro, étudiées par Anna Bochnakowa (2005) semblent manifester une filiation entre conservatisme politique et purisme linguistique ${ }^{5}$.

\section{3. «Le bon français » (Le Figaro, 1996 à 2006)}

Dans les chroniques de langue du Figaro, Pierre Bénard, Jacques Capelovici, Alain Feutry, Jean Dutourd, Bernard Leconte, Maurice Druon ${ }^{6}$ se relayaient pour attaquer anglicismes branchés, féminisation des titres et noms de métiers ou réforme de l'orthographe. L'académicien Jean Dutourd épinglait les modes langagières du moment, comme par exemple dans le billet du 29 mai 1999, qui relève j'hallucine pour je m'étonne, mon ego pour ma personne, ou maltraitance pour mauvais traitements: on peut lire dans les termes choisis l'expression d'un antagonisme droite/gauche : un agacement du vieux conservateur à l'égard d'un j'hallucine, imputable aux outrances de la jeunesse, à l'égard d'un ego, témoignant d'une appréhension psychanalysante, à laquelle la gauche est réputée encline, à l'égard d'une maltraitance trop "politiquement correcte »". Anna Bochnakowa, préférant structurer son ouvrage à partir d'un classement linguistique des mots commentés ("Prononciation », "Orthographe », «Morphologie », « Lexique », "Correction»), ne souhaite pas axer son analyse sur la dimension politique de ces chroniques ${ }^{8}$; elle ne peut se dispenser toutefois de consacrer un chapitre à la question de la féminisation (traitée sous la rubrique « Morphologie ») et souligne que «c'est une situation réelle, due à la politique d'un gouvernement comprenant des femmes qui a déclenché l'emploi de l'article féminin devant le mot ministre » (Bochnakowa, 2005 : 39). Ce gouvernement, c'est un gouvernement de gauche : celui que dirige Lionel Jospin de 1997 à 2002. La demande des ministres femmes du gouvernement, qui souhaitent se faire appeler « Madame la Ministre », déclenche

5. Bien sûr, la satire des mots à la mode n'est pas réservée à la presse de droite; au XXe siècle, les chroniques de Jacques Cellard dans Le Monde, de 1971 à 1985, témoignent par exemple de la présence du genre dans un journal situé au centre gauche. On notera cependant que des représentants particulièrement emblématiques de la lutte contre les mots à la mode, comme l'académicien Jean Dutourd ou Renaud Camus, fondateur du parti de l'In-nocence, se rangent politiquement dans un conservatisme de droite (voire d'extrême-droite, le programme du parti de l'In-nocence préconise par exemple de «réduire ou tarir les flux migratoires en direction de l'Europe et de la France », www.in-nocence.org, consulté le $1^{\text {er }}$ octobre 2014).

6. Pour ne citer que les chroniqueurs réguliers, cités par ordre décroissant du nombre de billets écrits (de 315 pour Pierre Bénard à 109 pour Maurice Druon pendant la période 1996-2000 explorée par Anna Bochnakowa (2005 : 99).

7. La notion de "politiquement correct », qui prend sa source dans les milieux universitaires américains et a fonctionné d'abord aux États-Unis comme une auto-désignation par la gauche de son propre discours, a été utilisé par les républicains américains, puis par la droite française pour stigmatiser une « police morale de la langue » (Furet, Nouvel Observateur, 29 août 1991, cité par Delporte, 2009 : 302).

8. "Nous éviterons les débats de cette nature [portant sur "les aspects sociaux et politiques"] tout en étant consciente non seulement de leur impact sur le discours relatif à la normativité dans l'enseignement mais aussi du rôle des institutions et des outils juridiques chargés par l'État de la codification et même de la protection du patrimoine national qu'est la langue française. » (Bochnakowa, $2005:$ 14) 
en septembre 1997 une polémique, dans laquelle les voix de droite, telles que celles de Maurice Druon et de Marc Fumaroli, s'élèvent contre cette nouvelle « mode». Fumaroli, que l'on peut qualifier d’« historien de droite » (Peigne-Giuly, 1995), présente en effet la féminisation comme un phénomène de mode:

L'offensive actuelle de féminisation des titres en France est en réalité une imitation tardive, provinciale, coloniale, sur les traces du Canada et de la Wallonie, d'une mode sectaire qui a eu son épicentre dans les universités des États-Unis. Son application à la langue française, dont les structures grammaticales sont moins costaudes que celles de l'anglais aurait quelque chose de caricatural. (Fumaroli, 1998: 1)

En adoptant les formes féminisées, les Français feraient donc preuve de suivisme à l'égard des ÉtatsUnis, dont le Canada et la Belgique seraient les premiers imitateurs, sans comprendre la catastrophe morphologique dans laquelle ils précipitent une langue française aux structures supposées « moins costaudes que celles de l'anglais ». Sur quoi l'historien fonde-t-il cette appréciation des structures syntaxiques du français ? II ne le dit pas. Le désir de stabilité pour la langue française sert ici à dénoncer des féminismes présentés comme des mots à la mode, avec un arrière-plan idéologique clairement politisé.

La chronique «Le bon français » perdure dans le même esprit jusqu'en 2006. Le 11 janvier 2006, Alain Feutry signe une chronique raillant la dénomination jeunes en déshérence pour désigner les jeunes en difficulté. La rédaction a-t-elle jugé cette satire trop « droitière »? En se moquant de la désespérance de la jeunesse pour préconiser un « désespoir roboratif », le chroniqueur serait-il allé trop loin? Toujours est-il que la chronique « Le bon français » est alors abandonnée en ce début 2006. Le Figaro la remplace par une chronique de nature différente, «Le mot du jour », qui consiste à choisir un mot en relation avec l'actualité du jour, et à en faire le prétexte d'un développement thématique et non linguistique. Les chroniques de langage disparaissent-elles alors?

\section{Chroniques de langage dans Libération et Le Monde : un récent passage à gauche}

Au cours des analyses de modalisateurs émergents que nous avons entrepris de mener, Gilles Siouffi, Chantal Wionet et moi-même (2011, 2012), nous nous sommes aperçus que les commentaires épilinguistiques sur les mots à la mode ne se trouvaient aujourd'hui pas tant dans Le Figaro que dans certains numéros d'été de Libération, et, depuis 2011, dans Le Monde.

\subsection{Libération : "Mots qui fâchent », "Expressions à la con »}

Pendant l'été2008, Libération ouvre à Anne Diatkine une chronique intitulée « Les mots qui fâchent», dont la durée de vie sera relativement limitée (du 15 juillet au 23 août 2008), mais dont l'apparition 
même dans un journal de gauche paraît révélatrice. La liste des mots traités par la journaliste permet de suivre comment elle cherche, et trouve son " genre ». Elle commence avec des mots ou expressions qui provoquent en elle une réaction d'agacement conforme à un certain purisme traditionnel, comme l'expression les gens (" oui, mais les gens ne comprendront pas ») qui lui déplaît par son caractère vague, ou le mot rapports (pour « rapports sexuels »), euphémisme bienséant. Mais elle en vient au repérage des modes langagières à partir de l'article " Pas d'amalgame », où l'on peut voir poindre une inflexion politique :

Ne nous demandons pas pourquoi les expulsions sont nommées «éloignements » par l'administration et ce que recouvre « l'invitation » à quitter le territoire français, et « l'harmonisation des flux ». Ce filtre des mots pour rendre invisible l'ignominie est un processus récurrent. Les techniciens nazis aussi employaient des mots propres, qui empêchaient de voir. On le note.

Avant toute possibilité de réflexion, on nous supplie : «Attention, pas d’amalgame! » Alors même qu'on tente de déconstruire et d'analyser, afin d'éviter de tout fondre, la réflexion est bloquée. Les entreprises sont « restructurées », quand elles licencient et « les ressources humaines » en sont ainsi « optimisées ». Nous nous sommes habitués à ce vocabulaire policé, censé volatiliser le réel, au point qu'il contamine les échanges les plus privés. Comment désigner ces mots écrans qui agissent comme des anesthésiants ou des drogues hallucinogènes? Tandis qu'à chaque grève, l'usager reste bien « pris en otage »! (Libération, 26 juillet 2008)

Avec « Pas d'amalgame », la journaliste ouvre la boîte de Pandore des euphémismes de la langue contemporaine. Elle épingle dès lors une série de mots utilisés dans le discours sur les politiques migratoires (éloignements, harmonisation des flux) ou dans le jargon d'entreprise (restructuré, ressources humaines, optimisé). Pour une journaliste de gauche, la dénonciation de ce qui relève, sinon des modes langagières, du moins d'un jargon moderne, clairement ancré dans une idéologie de droite, ne fait pas problème. Lancée sur cette voie, Anne Diatkine continue avec des expressions qui, cette fois, relèvent clairement de la mode langagière : de pas d'amalgame, déjà ancien, elle passe à pas de souci, le ressenti, c'est juste trop bien. À la fin de l'été, la "Chronique des mots qui fâchent » tourne court. Pourquoi? Sans doute fallait-il laisser place à l'actualité, plus pressante, de la rentrée ; peut-être aussi la journaliste ou sa rédaction ont-elles perçu un conflit entre ces chroniques satiriques et le style du journal lui-même. Anne Diatkine suspecte dans le pas de souci une zénitude quelque peu «baba cool »; elle subodore à propos du « ressenti » : « l'expression est peut-être née dans le sillage de 1968 » (30 juillet 2008). Elle se surprend elle-même à utiliser le juste intensifieur :

Il n'a même pas fallu un an pour qu'on soit des millions, plutôt jeunes, je crois, à ponctuer toutes nos appréciations du mot « juste ». C'est « juste » génial, c'était « juste » un cauchemar, tu peux « juste » pas savoir ce qu'il m'est arrivé, j'ai rencontré un mec « juste» trop bien. Moi aussi, je peux « juste » parler ainsi. (Libération, 22 août 2008) 
Dans la seule année 2008, pas de souci compte dans Libération 14 occurrences, le/du ressenti 15, et ses journalistes ne se privent pas d'utiliser juste comme intensifieur'. La satire des mots à la mode risque donc de se retourner contre l'idéologie post-soixantuitarde de Libération et sa pratique d'un parler «branché ». La chronique d'Anne Diatkine ne s'installe pas durablement.

À la faveur de l'accalmie estivale, des chroniques de langage ont cependant paru à nouveau dans Libération. Pendant l'été 2012, on a pu les lire sous la forme d'un bref feuilleton écrit à plusieurs mains $^{10}$, en six épisodes, publiés six jours d'affilée, du 20 au 25 août. Les chroniqueurs s'insurgent contre une invasion de la langue de l'entreprise, qui pratique et impose définir le périmètre, changer de logiciel, je reviens vers vous; ils reviennent vers pas de souci, devenu Y a pas de souci, pour saisir le paradoxe de son succès : « Notons d'ailleurs son regain depuis que la crise bancaire fait rage. "Y a pas de soucis", quand si, justement, tous azimuts, il y a beaucoup de soucis à se faire » (Lefort, Libération, 22 août 2012). Dans cette série, la corrélation entre idéologie de gauche et satire des mots à la mode semble s'être affirmée.

\subsection{Le Monde : "Juste un mot»}

C'est peut-être que, depuis 2011, un journaliste du Monde construit régulièrement, par sa chronique hebdomadaire, un ancrage au centre gauche de ce genre satirique. Didier Pourquery a commencé ses chroniques de langage, sous le titre "Juste un mot », en octobre 2011. Cette chronique perdure jusqu'à aujourd'hui ${ }^{11}$. On a ainsi pu constituer, à partir de la base de données Europresse un corpus de 84 chroniques de langage, parues pendant la période du 3 octobre 2011 au $1^{\text {er }}$ mars 2014.

Les premières chroniques portent sur des mots de circonstance, suscitant des développements d'actualité tels que « Forfait» (10 octobre 2011, à propos des téléphones portables) ou «Gentillesse » (14 novembre 2011, à propos de la « Journée de la gentillesse »). Mais assez rapidement, comme Anne Diatkine, Didier Pourquery découvre l'intérêt de s'amuser des mots à la mode et enchaîne: «Voilà » (9 janvier 2012), «Trublion » (16 janvier 2012), « S'inviter » (23 janvier 2012, comme dans « Le mariage homosexuel s'invite dans la campagne »), «Carrément! » (30 janvier 2012, comme dans ce dialogue : «- Tu viendras jeudi prochain? - Carrément! »), «Clivant» (6 février 2012), « Sur » (13 février 2012) $)^{12}$, sans s'interdire toutefois des retours à des articles plus thématiques comme « Libertin » (27 février 2012). L'exploration linguistique de Didier Pourquery s'étend maintenant sur une durée suffisamment longue pour nous permettre de décrire les groupes sociolinguistiques auxquels il impute

9. Ainsi dans « juste la plus belle découverte du millénaire » 25 septembre 2008), « juste une tuerie» (11 octobre 2008), « juste la fin du monde » (18 novembre 2008), « juste inoubliable (5 décembre 2008).

10. Bruno Icher, Gérard Lefort, Emmanuelle Peyret, Didier Péron, Philippe Lançon.

11. Elle paraît, depuis avril 2013, dans les pages du Monde «Magazine ».

12. " J'ai compris que c'était fichu avec cette petite annonce, lue sur Internet : un professeur de français y propose "des cours particuliers sur Bagneux"! » (Pourquery, Le Monde, 13 février 2012). 
les mots à la mode dont il se moque, et donc peut-être les implications politiques de ses billets satiriques.

Notons d'abord la trace dans les chroniques de Pourquery d'une forme de tradition du genre, par la présence de groupes qu'on y rencontre depuis les origines et qui sont réputés lancer les modes : les femmes et les jeunes. Les premières sont mentionnées chez Pourquery pour carrément:

Entendu dans le métro avant-hier : «Tu viendras jeudi prochain? - Carrément! » Les deux jeunes femmes qui s'expriment ainsi ont le regard clair, et tout montre chez elles cette tonicité, cette énergie que l'on trouve de nos jours chez les trentenaires « décidées ». (Le Monde, 20 janvier 2012)

Les seconds, désignés par les termes jeunes, jeunesse, ados, sont signalés comme de grands utilisateurs de j'avoue (9 juillet 2012), c'est abusé (20 août 2012), connu (dans « Regarde, elle est connue, non? », «Il est connu, son père? », 12 octobre 2013). Les uns comme les autres sont des personnages habituels de la satire linguistique depuis le XVII siècle, traditionnellement identifiés comme des lanceurs de mode. Il n'y a pas ici de spécificité idéologique dans le discours de Pourquery, mais une conformité au genre. Avec quatre mentions dans le corpus, les deux groupes ne sont finalement que rarement signalés.

Par sa profession, Pourquery est évidemment sensible aux modes du discours de presse, où il relève s'inviter:

Une consœur chroniqueuse au Monde me montre un communiqué de presse. Le texte commence par un alléchant: « Les rapports complexes entre médias et justice s'invitent à la table ronde de... » Vous l'avez remarqué : beaucoup de gens et de choses «s'invitent », ces derniers temps, dans l'actualité et ses titres. (Le Monde, 23 janvier 2012)

Le journaliste observe les usages auxquels sacrifient « ces derniers temps » ses consœurs et confrères. Il relève de même chez eux le tour De quoi X est-il le nom ? (5 octobre 2013, d'après le titre du livre d'Alain Badiou De quoi Sarkozy est-il le nom ? [2007], les journalistes forment « De quoi Cahuzac est-il le nom ? »). L'observation de ce groupe socioprofessionnel est imputable à la proximité de l'observateur, et ne paraît pas entrer dans un discours politiquement orienté.

Mais le groupe qui est majoritairement pointé au fil de ses chroniques, c'est le monde de l'entreprise et de la communication. C'est à eux, selon Pourquery, que sont imputables : deadline (4 mai 2013), un ptit mail (15 octobre 2012), dispo (3 décembre 2012), naming (21 janvier 2013), en charge de (2 juillet 2012), soutenabilité (28 janvier 2013), overbookée (11 février 2013), busy (11 février 2013), dans la boucle (1 $1^{\text {er }}$ mars 2014) $)^{13}$. Les managers et communicants en tout genre apparaissent comme les

13. «L'obsession des gens aujourd'hui dans les entreprises : être dans la boucle [...]. Bien sûr, à première vue, la boucle est tout simplement la liste des destinataires directs ou « en copie » de nos courriels d'information ou de convocation aux

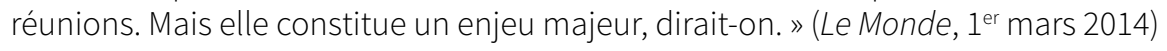


sources de ces mots relayés dans la langue commune ${ }^{14}$. Mais qui sont, selon Pourquery, les usagers et diffuseurs de ces mots?

Les indications sociologiques précises, rares, situent les locuteurs dans les classes moyennes: infirmières (16 avril 2012), employés de bureau (16 avril 2012), « cadres quadra » (18 janvier 2014). C'est surtout par leur espace conversationnel que Pourquery saisit la sphère sociale où il observe le passage des mots à la mode dans la «langue commune» (Branca-Rosoff, 2011 : 7-26). Les lieux privilégiés pour l'observation de la diffusion ne sont plus, comme dans les satires de François de Callières, les salons de la noblesse ; il ne s'agit pas non plus, comme dans celles de l'abbé Royou, des clubs politiques. Didier Pourquery cherche à saisir la langue commune dans les bureaux (15 octobre 2012 ; 11 février 2013 ; 4 mai 2013), et, surtout, dans les rames du métro ${ }^{15}$. La mention de cet espace linguistique est récurrente dans ses chroniques:

V'là, oualà, voualà,'là, selon la prononciation et l'intensité qu'on lui donne : ici ou là, il parsème nos phrases comme un nouveau tic de langage. C'est le chef du service Culture du Monde qui me l'a fait remarquer. Il sait ce dont il parle, les mots, ça le connaît. Alors, depuis, j'écoute les dialogues dans le métro, à la cafétéria, en réunion, à l'affût du fameux voilà. (Le Monde, 9 janvier 2012)

Entendu dans le métro avant-hier : «Tu viendras jeudi prochain ? - Carrément! » (Le Monde, 20 janvier 2012)

Trois phrases entendues dans le métro cette semaine m'ont fait dresser l'oreille. D’abord lundi : «Attends, j’t'assure, c'est juste le meilleur resto de viande de Paris », déclarait un jeune homme bien mis à son collègue de bureau en lui parlant de sa découverte du week-end. (Le Monde, 16 avril 2012)

Depuis six ou sept ans, son utilisation dans mes chères conversations de métro a explosé : "Ce matin, je suis en mode radar », «sur ce coup, j’suis en mode veille », « arrête, j’suis en mode vénère ». (Le Monde, 2 juillet 2012)

14. Pourquery note l'influence de l'informatique, par sa terminologie avec en mode (Le Monde, 2 juillet 2012), data (24 décembre 2012) et par son fonctionnement binaire avec ou pas (4 mars 2013). Le vocabulaire de l'informatique n'est pas intrinsèquement lié au monde de l'entreprise. Il est cependant, dans le fonctionnement des sociétés postmodernes, fortement lié à une conception entrepreneuriale du monde du travail (Isnards et Zuber, 2008).

15. Dans une chronique récente, Didier Pourquery cherche à suivre le chemin de la diffusion de la locution à la mode se faire un p'tit billet. Il la repère sur un forum de discussion à propos de la taxation des heures supplémentaires en 2007, elle est reprise dans le monde de la finance, puis du commerce : « Au départ c'est le résultat d'une transaction directe, de la main à la main, de l'"économie informelle" comme on dit à Bercy. Puis les commerciaux adoptent le terme qui se répand dans les entreprises » (Le Monde, 18 janvier 2014). Il l'entend dans le TGV, prononcée par deux « cadres quadra », tout en soulignant: « Je l'entends souvent dans d'autres contextes (métro plutôt) » (18 janvier 2014). Selon Pourquery, le métro apparaît comme le lieu où se parle la « langue commune ». 
J'avoue, je suis crevé. J'avoue tout ce que vous voulez. Lâchez-moi un peu. Mes voisins de métro sont de plus en plus souvent dans cet état d'esprit. Les «j'avoue » crépitent en sourdine entre les barres nickelées et les strapontins malcommodes. (Le Monde, 9 juillet 2012)

Voilà bien cinq ans que cette expression [ c'est abusé »] résonne à la sortie des collèges et que je l'entends dans les rames de métro. J'ai fait mon enquête. Sur le moteur de recherche Internet le plus utilisé, «c'est abusé » affiche plus de 30 millions d'occurrences. (Le Monde, 20 août 2012)

Le plus horripilant dans ce que j'entends dans les bureaux ou les métros est «j'suis overbookée », overbooked comme les avions ou les mannequins dans les agences! Ou pire "j'suis busy» (il existe d'ailleurs un blog girly à l'enseigne « So Busy Girls»). (Le Monde, 11 février 2013)

Cette semaine, la France a vécu un épisode neigeux. Enfin pas toute la France, mais la région parisienne et les plages du week-end des Parisiens étant notamment sous la neige, on peut dire que la France a vécu un épisode neigeux. Parlez-en à mes codétenus de la ligne 6 du métro, ils vous raconteront cet épisode, dont ils furent les « galériens ». (Le Monde, 18 mars 2013)

C'est par la description de l'espace conversationnel que Pourquery caractérise la situation sociale des locuteurs. La citation du 9 juillet 2013 décrit des conditions de vie pénibles : crépitent et barres nickelées restituent un univers sonore, avec les désagréments de ses bruits répétitifs et métalliques; strapontins malcommodes rappelle l'inconfort des sièges du métro, inconfort qui sera ressenti d'autant plus douloureusement par l'usager qu'il s'avoue d'emblée «crevé ». La dernière citation, datée du 18 mars 2013, choisit, pour ce groupe social, des dénominations qui reprennent, sur le mode hyperbolique, des sèmes négatifs : la pénibilité du travail avec le mot galériens, et celle de l'enfermement dans la routine comme dans la rame de métro, avec le mot codétenus. La représentation que donne Pourquery des diffuseurs de mots à la mode n'aurait rien à envier à celle des mineurs de fond de Germinal, si ce n'était la pointe d'humour qu'il glisse, par la caricature, dans son billet.

Les usagers et diffuseurs des mots à la mode apparaissent ainsi sous les traits d'une classe opprimée et soumise, victime inconsciente d'une novlangue dont entrepreneurs et communicants sont les initiateurs. La satire des mots à la mode entre ainsi dans un schéma inverse de celui de la fin du XXe siècle: ce n'est plus la droite qui reproche à la gauche d'abuser d'un jargon à la mode, mais la gauche qui détecte et raille un jargon à la mode initié par la droite.

\section{3. « Changement de paradigme»?}

Ce changement d'orientation politique des chroniques de presse correspond à l'apparition de pamphlets sur le «new wording » d'entreprise, dénoncé par Thomas Zuber et Alexandre des Isnards dans L'Openspace m'a tuer (2008), puis sur les usages, notamment linguistiques, des réseaux linguistiques dans Facebook m'a tuer (2011), tandis que Zoé Shepard signale la diffusion de cette novlangue au 
sein même du secteur public dans Absolument dé-bor-dée (2010), puis Ta carrière est fi-nie (2012) ${ }^{16}$. La satire - ou l'analyse engagée - des mots à la mode apparaît sinon passée à gauche, du moins pratiquée activement par la gauche.

\section{Conclusion}

Comment concilier cette pratique fondamentalement conservatrice avec le mot d'ordre « changement d'abord! » que Michel Winock donnait pour définitoire de la gauche ? Sans doute faut-il replacer l'évolution de la satire linguistique dans les changements politiques récents qu'ont connus la droite, et par contrecoup, la gauche; l'élection de Nicolas Sarkozy en 2007 a bousculé une certaine caractérisation de la droite, particulièrement sur le critère du changement: le mot d'ordre "Stabilité d'abord! », que Michel Winock utilisait pour caractériser la droite, peut-il ici convenir ? Avec Nicolas Sarkozy, la droite est entrée dans une «stratégie du mouvement perpétuel » (Winock, $2012: 189)^{17}$. D’où entre droite et gauche un brouillage des lignes, que Michel Winock explicite :

La mondialisation libérale, à laquelle la droite souhaite adapter notre société, c'est le développement du consumérisme, le règne de la « pub », l'escalade de la violence et du sexe sur les chaînes de télé en quête d'audience, l'écriture phonétique des SMS, le goût de l'épanouissement personnel au détriment du culte de la patrie : bref, la droite gouvernante promeut un système économique qui assure le triomphe de l'individualisme que, dans le même temps, elle condamne sans appel au nom des valeurs qu'elle tient pour les plus hautes, celles de la morale républicaine, de la grandeur nationale ou des idéaux spirituels.

Symétriquement, la gauche adhère à la révolution des mœurs, à la remise en cause des hiérarchies, au relativisme des valeurs que favorisent l'économie et la culture mondialisées, tout en s'opposant à celles-ci beaucoup plus souvent et fortement que la droite. (Winock, 2012: 220-221)

Par l'adhésion à la «mondialisation libérale », et le « new wording » qui l'accompagne, par la «stratégie du mouvement perpétuel », le discours de droite entraîne la langue dans une farandole de mots à la mode, dans laquelle les sujets de langue se trouvent pris; ce contexte peut expliquer l'émergence à gauche, par réaction, d'une satire politique des mots à la mode.

16. Les universitaires s'intéressent depuis une vingtaine d'année à la diffusion des usages linguistiques d'une classe dominante, sous l'étiquette de « langue de bois » (Tournier, 1986 ; Delporte, 2009), ou de « langue de coton » (Huygue, 1991). Récemment, des analyses ont été consacrées à la diffusion des mots du libéralisme dans le secteur universitaire lui-même (Monte, 2013).

17. La stratégie du mouvement n'est pas sans racine dans l'histoire de la droite, en particulier dans celle de la droite que René Rémond catégorisait comme «bonapartiste », et le conservatisme s'est, dès la période révolutionnaire, interrogé sur ses propres apories (Slama, 2006 [1992] : 813-814). 
Alors que la droite représentait le conservatisme social et, en particulier, le conformisme linguistique, une inversion de tendance se fait jour en ce XXI ${ }^{e}$ siècle commençant. Dans le bilan qu'il dressait fin 2012, Didier Pourquery la décrivait ainsi :

Sur le front du langage, 2012 est coincée entre les mots d'une droite décomplexée et ceux d'une gauche qui ne l'est pas vraiment. Cela s'accompagne de conformismes divers et de litotes politiquement correctes; le retour de ce qu'il faut dire ou ne pas dire (le retour du « Non, tu ne peux pas dire ça »); le « mariage pour tous » à la place du « mariage gay »; la mode d'un certain vocabulaire bien-pensant avec son lot de « lien social », de «vivre-ensemble » et ce genre de phrases toutes faites. (Le Monde, 31 décembre 2012)

La satire des travers langagiers de la gauche par la gauche n'est pas neuve: dès l'époque révolutionnaire, la gauche pratique l'autodérision. Ce qui étonne davantage dans la période contemporaine, c'est que l'impulsion des modes langagières vienne de la droite, et que ce soit désormais la gauche qui s'empare du fouet de la satire pour stigmatiser les mots à la mode. C'était à l'origine la presse contre-révolutionnaire qui dénonçait un abus de mots à la mode qu'elle imputait à la gauche; c'est à présent dans la presse de gauche et de centre gauche que se déploie une satire de mots à la mode imaginés par une droite entrée dans une «stratégie de mouvement ». Assistons-nous là, pour reprendre le mot à la mode épinglé par Didier Pourquery dans sa dernière chronique, à un « changement de paradigme » (Le Monde, 26 octobre 2013) ? Avançons du moins que les systèmes de clivages qu'organise la satire des mots à la mode sont complexifiés par la possibilité d'un anticonformisme de droite : en plus du clivage sociologique interne des origines, en plus d'un antagonisme politique orienté droite contre gauche, mis en place avec la Révolution, il faut compter aussi avec un antagonisme politique orienté gauche contre droite. 


\section{Références}

\section{Sources primaires}

Badiou, Alain (2007), De quoi Sarkozy est-il le nom ?, Paris, Nouvelles Editions Lignes.

Boursault, Edme (1694), Les Mots à la mode, en un acte, en vers, Paris, Didot.

Callières, François de (1692), Des mots à la mode et des nouvelles façons de parler, avec des observations sur diverses manières d'agir et de s'exprimer, et un discours en vers sur les mêmes matières, Paris, Barbin.

Camus, Renaud, Parti de l'In-nocence, disponible sur http://www.in-nocence.org/index.php?page=programme\&prog=immigration. [Page consultée le 10 mars 2014.]

Chantreau, Pierre-Nicolas (2008 [1790]), Dictionnaire national et anecdotique, édition présentée et annotée par Agnès Steuckardt, Limoges, Lambert-Lucas.

Desfontaines, Pierre-Guyot (1726), Dictionnaire néologique, [s. l.], [s. n.].

Diatkine, Anne (2008), « Les mots qui fâchent », chronique de langage, Libération, juillet-août.

Dutourd, Jean, Pierre Bénard, Jacques Capelovici, Alain Feutry, Bernard Leconte et Maurice Druon (1996-2006), «Le bon français », chronique de langage, Le Figaro.

Estienne, Henri (1579), Deux dialogues du nouveau langage françois, italianizé, \& autrement desguizé, principalement entre les courtisans de ce temps, Anvers, G. Niergue. [1éd., 1578.]

Fumaroli, Marc (1998), «La querelle du neutre », Le Monde, 31 juillet 1998, p. 1.

Grand Corpus des grammaires, remarques et traités de langue (XIVe-XVIIe siècles), sous la direction de Bernard Colombat, Jean-Marie Fournier et Wendy Ayres-Bennett, Paris, Classiques Garnier numérique.

Isnards, Alexandre des et Thomas Zuber (2008), L'Openspace m'a tuer, Paris, Hachette Littératures.

Isnards, Alexandre des et Thomas Zuber (2011), Facebook m’a tuer, Paris, NIL éditions.

Juvénal (1965 [I'e siècle ap. J.-C.]), Satires III, IV, V, édition de René Marache, Paris, Presses Universitaires de France.

Les Actes des Apôtres (1789-1791), Paris, [s. n.].

Micromégas [Antoine Cérutti] [1789], Prospectus d'un dictionnaire d'exagération, destiné à MM. Les Rédacteurs du journal de Paris, [s. l.], [n. d.].

Pourquery, Didier (2011-2013), « Juste un mot », Le Monde (Le Monde Magazine à partir d'avril 2013).

Royou, Thomas-Marie, abbé (1790), L’année littéraire et politique. 
Shepard, Zoé (2010), Absolument dé-bor-dée, Paris, Albin Michel.

Shepard, Zoé (2012), Ta carrière est fi-nie, Paris, Albin Michel.

Vaugelas, Claude Favre de (1690), Nouvelles remarques de M. de Vaugelas sur la langue françoise, Paris, Guillaume Desprez.

\section{Sources citées}

Baecque, Antoine de (2000), Les éclats du rire : la culture des rieurs au XVIIIe siècle, Paris, Calmann-Lévy.

Bochnakowa, Anna (2005), Le «bon français » de la fin du XXe siècle : chroniques du Figaro (1996-2000), Cracovie, Publications de l'Université Jagellone.

Branca-Rosoff, Sonia (2011), «Langue prescrite, normativités, changements de norme », dans Sonia Branca-Rosoff, Jean-Marie Fournier, Yana Grinshpun et Anne Régent-Susini (dir.), Langue commune et changements de normes, Paris, Champion, p. 7-26.

Brunot, Ferdinand (1927), Histoire de la langue française des origines à 1900, tome 9 (La Révolution et l'Empire), Paris, Armand Colin.

Delporte, Christian (2009), Une histoire de la langue de bois, Paris, Flammarion.

Griffe, Michel (2001), «Auctoritas verborum : la linguistique normative à l'époque impériale », dans Gilles Siouffi et Agnès Steuckardt (dir.), La norme lexicale, Montpellier, Publications de l'Université Paul-Valéry Montpellier 3, p. 131-156.

Guilhaumou, Jacques (2009), « Modérer la langue politique à l'extrême : les journalistes remarqueurs au début de la Révolution française », Annales Historiques de la Révolution française, n³, p. 21-46.

Huygue, François-Bernard (1991), La langue de coton, Paris, Robert Laffont.

Merlin-Kajman, Hélène (à paraître), « Mode et mimétisme dans Les mots à la mode de François de Callières et quelques comédies », dans Gilles Siouffi (dir.), Modes langagières dans l'histoire, Paris, Champion.

Monte, Michèle (dir.) (2013), Les discours sur l'enseignement supérieur et la recherche, numéro thématique de la revue Mots : les langages du politique, nº 103.

Peigne-Giuly, Annick (1995), « Le débat culturel sans question », Libération, 20 mars 1995, p. 32.

Rémond, René (1982), Les droites en France, Paris, Aubier.

Ricken, Ulrich (1982), «Réflexions du XVIIIe siècle sur l’abus des mots », Mots, n 4, p. 29-45.

Siouffi, Gilles, Agnès Steuckardt et Chantal Wionet (2011), «La modalisation de l'énoncé à l'écrit: enquêtes sur les représentations et les usages. Le cas de c'est vrai que », L'information grammaticale, $n^{\circ} 130$, p. 11-17. 
Siouffi, Gilles, Agnès Steuckardt et Chantal Wionet (2012), "Comment enquêter sur les diachronies courtes et contemporaines? ", dans Franck Neveu, Valelia Muni Toke, Peter Blumenthal, Thomas Klingler, Pierluigi Ligas, Sophie Prévost et Sandra Teston-Bonnard (dir.), Actes du $3^{e}$ Congrès mondial de linguistique française, Lyon, France, 4-7 juillet, Les Ulis, EDP Sciences, p. 215-226, disponible sur http://dx.doi.org/10.1051/shsconf/20120100214. [Page consultée le 11 mai 2015.]

Siouffi, Gilles, Agnès Steuckardt et Chantal Wionet (2014), «Le mot à la mode : usage et enjeu d'une expression métalinguistique profane », Le discours et la langue : revue de linguistique française, tome 6.1 (Métalangage et expression du sentiment linguistique "profane », sous la dir. de Michelle Lecolle), p. 127-141.

Slama, Alain-Gérard (2006), « Portrait de l'homme de droite: littérature et politique », dans Jean-François Sirinelli (dir.), Histoire des droites en France, tome 3 (Sensibilités), Paris, Gallimard, p. 787-838. [1 éd., 1992.]

Steuckardt, Agnès (2001), «L'abus des mots, des Lumières à la Révolution », dans Gilles Siouffi et Agnès Steuckardt (dir.), La norme lexicale, Montpellier, Publications de l'Université Paul Valéry, p. 177-196.

Steuckardt, Agnès (2008), «Introduction », dans Pierre-Nicolas Chantreau (2008 [1790]), Dictionnaire national et anecdotique, édition présentée et annotée par Agnès Steuckardt, Limoges, Lambert-Lucas, p. 9-91.

Steuckardt, Agnès (2011), « Le Dictionnaire néologique de l'Abbé Desfontaines ou la résistance à l'innovation linguistique », dans Stéphanie Génand et Claudine Poulouin (dir.), Entre deux mondes: les frontières du $18^{e}$ siècle, Paris, Desjonquères, p. 36-48.

Tournier, Maurice (1986), «Critique de la critique : langue de bois et parler vrai », Mots, n 13, p. 191194.

Winock, Michel (2012), La droite hier et aujourd'hui, Paris, Perrin. 